REVISTA CHILENA DE LITERATURA

Noviembre 2019, Número 100, 171-190

\title{
LA REVISTA CHILENA DE LITERATURA EN CUATRO MOMENTOS DE SU INTERNACIONALIZACIÓN
}

\author{
Horst Nitschack \\ Universidad de Chile \\ Santiago de Chile, Chile \\ horst.nitschack@gmail.com \\ Irmtrud König \\ Universidad de Chile \\ Santiago de Chile, Chile \\ Irmtrud.konig@gmail.com
}

RESUMEN / ABSTRACT

Este artículo propone una lectura del proceso de internacionalización de la Revista Chilena de Literatura $(R C h L)$, es decir, su apertura hacia otras literaturas del mundo y su potencial crítico en el marco de las Humanidades, desde su fundación en el año 1970 hasta el número 99 de 2019. Este recorrido se focaliza, por razones metodológicas, en cuatro momentos particularmente significativos de este proceso en tanto se vinculan -directa o indirectamente- a la cambiante realidad histórica chilena. En este marco general, el artículo se detiene, en primera instancia, en los años desde su fundación y el periodo de la Unidad Popular, caracterizables por su posicionamiento en el debate académico nacional con un marcado acento en la literatura chilena y su inserción en Latinoamérica. Este perfil se modifica drásticamente bajo el impacto del golpe militar y los silenciamientos impuestos durante casi dos décadas de dictadura, probablemente el periodo más decantadamente internacional y "neutral" en términos temáticos, metodológicos e ideológicos, pero no carente de un importante potencial "humanista" universal. Con la transición a la democracia, el perfil de la revista se abre no solo a distintos ámbitos de la literatura mundial, tendencias y perspectivas teórico-metodológicas, sino que evidencia una paulatina pero sostenida apertura hacia temas, obras y autores vinculados al testimonio y la memoria del pasado reciente. Finalmente, el artículo, examina los últimos decenios de la revista bajo el prisma de los impactos de la era digital, tanto en su "materialidad" electrónica como su potencial humanista sobre el horizonte de un proceso de internacionalización cada vez más global, del que la $R C h L$ no permanece al margen y en el que renueva y consolida sus compromisos con la vida literaria chilena, sin renunciar al horizonte internacional conquistado. 
PALABRAS ClaVe: revistas literarias, literatura chilena y latinoamericana, literaturas del mundo, literatura y humanidades, crítica literaria.

\section{THE REVISTA CHILENA DE LITERATURA IN FOUR MOMENTS OF ITS INTERNATIONALIZATION}

This article proposes a reading of the internationalization process of $R C h L$ from its foundation in 1970 to the issue 99 of 2019, that is, its opening to other literatures of the world and its critical potential in the framework of the Humanities. In methodological terms, this study focuses on four significant moments of this process which are linked-directly or indirectly - to the changing reality of Chilean history. In this general framework, the article first examines the beginnings of RChL in the period of the Unidad Popular. During this period, the publication participates in the national academic debate with emphasis on Chilean literature and its insertion in Latin America. This profile is dramatically modified with the impact of the military coup and the silence imposed during almost two decades of dictatorship. However, this is probably the period of the most international and at the same time "neutral" tendency in thematic, methodological, and ideological terms, yet bearing always an important "humanist" potential. With the transition to democracy, the profile of the magazine opens not only to different areas of world literature, trends and theoretical-methodological perspectives, but also shows a gradual but sustained openness towards themes, works and authors linked to the testimony and the memory of the recent past. Finally, the article examines the last decades of the magazine under the lens of the impact of the digital era, both in its electronic "materiality" and its humanistic potential in the horizon of an increasingly global internationalization process. While it renews and consolidates its commitment to Chilean literary manifestations, $R C h L$ also strengthens its connection to international horizons.

KEYWORDS: Literary magazines, Chilean and Latin American literature, literatures of the world, literature and humanities, Literary criticism.

Recepción: 08/05/2019 Aprobación: 21/06/2019

La $R C h L$ fue fundada, su título lo anuncia, como una revista de Literatura, esto es, una revista de literatura publicada en Chile, vinculada a una de las disciplinas cultivadas en la Facultad de Filosofía y Humanidades de la Universidad de Chile, los estudios literarios. Tanto el título de la revista como el lugar de su publicación dejan entrever una tensión que atraviesa toda la revista de una manera creativa, pero también como un desafío permanente: la tensión entre lo nacional -Chile- y lo internacional, la tensión entre lo local y lo universal. 'Literatura' y 'Facultad de Filosofía y Humanidades' constituyen referentes que implican en ambos casos un contexto histórico específico, pero al mismo tiempo una conceptualización universal, pensados, como se especifica también en el título,-'Revista chilena' y el nombre de la Universidad-, desde Chile y mirando a Chile. En consecuencia, esta revista no 
se puede examinar sin tener presente que sus artículos han sido publicados-y en muchos casos también fueron escritos- en este país y, por lo tanto, que como lectores estemos conscientes de este hecho y de que cada publicación fue aprobada por un Comité de Redacción integrado por académicos del Departamento de Literatura de esta Universidad.

Por otra parte, cabe constatar que, junto a los estudios sobre literatura chilena, la revista contiene una cantidad considerable de artículos que se refieren a literaturas latinoamericanas y del Caribe y a literaturas europeas. En cambio, comparativamente es mucho menor el número de trabajos que se dedican a la literatura de los Estados Unidos y de Canadá y ninguno se refiera a literaturas de Asia, África o Australia. En este sentido se repite y se consolida un concepto de literatura en el cual prevalece la tradición europea, aunque probablemente no se trata de una opción conscientemente asumida por sus editores. No obstante, hay un desplazamiento importante en su enunciación: se trata de miradas sobre la literatura articuladas, en su gran mayoría desde Chile y América Latina y si este no es el caso, -ya lo hemos mencionado-de todos modos son artículos que un comité de redacción chileno ha seleccionado para participar en un diálogo que se proyecta desde este país. En este sentido podemos entender la $R C h L$ también como documento del modo cómo se construye la literatura mundial desde Chile.

Al mismo tiempo, y este es otro aspecto que nos interesa destacar en este ensayo, la historia de la $R C h L$ refleja también la historia de las Humanidades en Chile durante los últimos cincuenta años. Refleja sus expectativas, sus desafíos y amenazas, pero también su rol en un mundo marcado por nuevas tecnologías, por el triunfo de un modelo económico (el neoliberalismo) y una globalización orientada a la maximización de los lucros financieros y las consecuencias ya conocidas: escasez de los recursos naturales (tierras de cultivo, agua), aumento de la contaminación y aceleración del cambio climático, nuevas desigualdades a nivel mundial y sus consecuencias: las migraciones. Ante este panorama amenazante, las Humanidades están llamadas a cumplir una función de observador crítico y aun más: asumir una posición y práctica de resistencia. Considerando que las artes, la literatura y las manifestaciones culturales en general -en tanto objetos privilegiados de las Humanidades-ya se han posicionado críticamente ante estos procesos, a los estudios sobre literatura les correspondería asumir también este potencial crítico, es decir, consignarlo, analizarlo, comentarlo e interpretarlo. Pero ello no es siempre evidente, como demuestra también la historia de esta revista: los estudios literarios pueden ser neutralizados y las obras pueden 
ser sometidas a perspectivismos que se interesan por cuestiones netamente filológicas, estructurales, de teoría literaria muy generales, sin ninguna referencia a las circunstancias históricas, sociológicas y políticas concretas en las cuales se inscriben.

La $R C h L$ fue fundada en el año 1970 durante el gobierno de la Unidad Popular, en una fase histórica en la cual el optimismo de los años 60 aún estaba vigente. Sin embargo, si lo vemos desde la perspectiva de nuestra distancia histórica, ya en esa época se vislumbraban cambios y reorientaciones cuyas consecuencias radicales vivimos en la actualidad en la medida en que las tentativas de transformaciones político-económicas profundas a favor de una sociedad más igual y humana son reemplazadas por los programas de modernización técnica, de la extensión de los mercados y de la intensificación de las libertades individuales. En otras palabras, ya se vislumbra la derrota de los proyectos políticos estimulados por el optimismo socialista. El proyecto 'Cuba' queda bloqueado internacionalmente y en la propia isla, los resultados más llamativos del mayo de París implicaron innovaciones en la moda y de la vida de consumo, pero no en la realidad de los procesos de producción y la organización económica y financiera de las sociedades industrializadas. En Praga, en el 68, el socialismo real mostró su potencial represivo. Al mismo tiempo se realiza en 1969 la llegada del primer ser humano a la luna, un evento simbólico con el cual se anuncia el perfeccionamiento tecnológico y el triunfo de un sistema económico-financiero que se expandirá globalmente y que se consolidará con el derrumbe del 'socialismo real' a partir de 1989.

En Chile, la Unidad Popular y el último gobierno socialista elegido por voto popular en un país de cultura occidental serán derrotados por la dictadura militar en 1973. Para la $R C h L$, en ese momento con seis números publicados, esto tiene como consecuencia su suspensión por tres años. En 1976 sale el número 7 de la revista, sin una palabra explicativa sobre las razones de su interrupción, aunque la obviedad de la situación no parece necesitar de comentarios o explicaciones. No obstante, encontramos en la página de los datos editoriales de este número una innovación bajo la rúbrica 'Colaboraciones' en nada comparable a las indicaciones correspondientes que aparecen en los números que fueron publicados durante los años de la Unidad Popular: "Solo se aceptan las colaboraciones solicitadas. Estas deben remitirse al Director". No sabemos, ¿solicitadas por quién? Probablemente por el propio Director o por el Comité de Redacción, que en este momento estaba constituido por cuatro profesores y una profesora del "Departamento de Literatura” (no más 'Departamento de Español' como decía en el número 
5/6). Este requisito de colaboración va a mantenerse durante toda la década de los 80, es decir, durante todos los años de la dictadura militar y se mencionará por última vez en el número 36 del mes de noviembre de 1990. A partir del número 37 (abril 1991) leemos: "Las colaboraciones deben remitirse al Director, por duplicado y con una síntesis de su contenido (Abstract)". Es decir, solamente a partir de 1991 se abre la posibilidad de que cualquier autor/a, sin ser invitado/a expresamente pueda presentar una colaboración. A partir del número 49 (noviembre 1996), una indicación adicional nos deja constatar que la revista está entrando en la época de los textos digitalizados. Se complementa la indicación de 'Colaboración' con la información adicional: "acompañar diskette WP".

Un cambio significativo en las políticas y las prácticas de publicación que refleja además la democratización y también la internacionalización, lo constatamos entre el número 56 (abril 2000) y el número 57 (noviembre 2000), con la siguiente rúbrica: "Las colaboraciones deben remitirse por duplicado, con una síntesis de su contenido (Abstract) y con su respectivo disquete. Referencias críticas según MLA, 1999”. Se renuncia a la instancia del director como figura central en la recepción de los artículos y se establece el formato de las citas y la bibliografía según el estándar internacional de la Modern Language Association.

Pero, ¿cómo partió la revista en 1970 en tiempos de la Unidad Popular? De los seis artículos publicados en el primer número, cinco son estudios sobre temas literarios: el artículo del fundador de la revista, Cedomil Goic, sobre "La Araucana", un trabajo de Carlos Santander T. sobre Jorge Luis Borges, un estudio de Ana María Sanhueza sobre Rayuela de Cortázar y los artículos de Antonio Skármeta y de Ariel Dorfman sobre literatura chilena, el primero sobre el drama chileno a inicios del siglo XX, y el segundo sobre dos autores chilenos cuya divergencia era ya evidente en esa época para el autor: Jorge Edwards y Antonio Skármeta. Hoy, casi cincuenta años más tarde, solamente se puede confirmar esta evaluación. El sexto artículo corresponde a un conjunto de poemas de Nicanor Parra: Palabras obscenas. En dos de estos poemas se vislumbra el dilema de esta generación de intelectuales y poetas chilenos: la identificación con el Chile de la Unidad Popular, y la coherencia que exige su compromiso político para sus relaciones con el mundo occidental, el mundo capitalista, así como también la solidaridad inexcusable con el país que en estos años sufrió la agresión estadounidense: Vietnam. 


\title{
SALTA A LA VISTA
}

que no debiera venir a los EE. UU.

-es comulgar con ruedas de carreta-

claro que por la misma razón

habría que romper relaciones con Francia

con Perú con Bolivia con Luxemburgo

no debiera moverme de Chile

pero quién engordaría con eso.

(RChL 1, 81).

\author{
MY STOMACH \\ may be in this country \\ but \\ my \\ heart \\ is \\ in VIETNAM \\ (RChL 1, 81).
}

Por otra parte, el texto publicado en la sección 'Notas' de este primer número, escrito por Mario Rodríguez Fernández, está dedicado a la poesía de Mario Benedetti. Como en el caso de las poesías de Nicanor Parra, son versos escritos desde América Latina, en este caso haciendo alusión a las batallas y victorias de los aliados en la Segunda Guerra Mundial y al lanzamiento de la bomba atómica sobre Hiroshima. El título debe ser leído como clara ironía: la "Imitación de Propercio", cuyos versos de ningún modo 'imitan' los líricos elogios a la amada Cintia del poeta romano (s. I a.C.), o su descripción de la batalla naval de Accio (Elegías, Libro IV); más bien constituyen un distanciamiento radical tanto de esta poesía como de los eventos a los cuales se hace mención en estos versos.

\author{
IMITACIÓN DE PROPERCIO \\ Yo no canto la defensa de Stalingrado \\ ni la campaña de Egipto \\ ni el desembarco de Sicilia \\ ni la cruzada del Rhin del general Eisenhower: \\ y llovió del cielo Estronsium 90 \\ Cesium 137 \\ Carbono 14
}




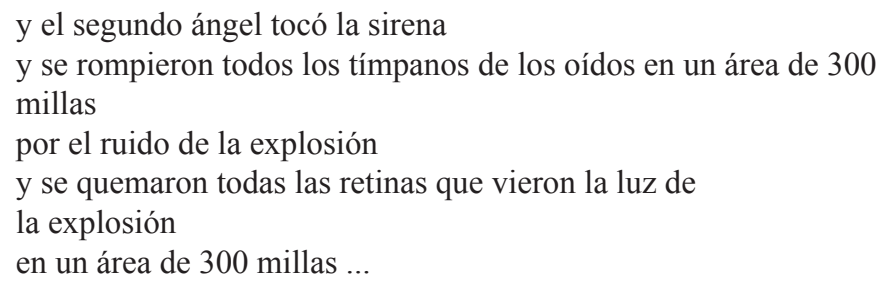

(Fragmento de Apocalipsis) (RChL 1, 87-88).

Estos ejemplos, como los que siguen, hacen patente que en este primer número se abre un horizonte hacia la literatura latinoamericana que se enuncia desde Chile y con plena conciencia del lugar de su escritura en el mundo. Este planteamiento vale también para la novela Rayuela de Julio Cortázar, que discute -aunque sin explicitar esta perspectiva-el artículo de Ana María Sanhueza: esta novela, escrita en París y situada en su primera parte en esta ciudad, se abre a través de sus múltiples alusiones a la cultura europea, pero es al mismo tiempo una de las novelas fundacionales del boom latinoamericano y de la renovación de la literatura occidental desde América Latina.

Al comparar el último volumen de 1972, que reúne los números 5 y 6, con el primer número que aparecerá en el año 1976 durante la dictadura militar, podemos constatar un cambio evidente. El número 5/6 estaba focalizado en la literatura hispanoamericana contemporánea con cinco artículos sobre literatura rioplatense, dos artículos sobre Gabriel García Márquez y dos artículos sobre Ernesto Cardenal. En la sección 'Notas' se recogen un trabajo del autor cubano Roberto Fernández Retamar, sobre el crítico literario, también cubano, José Antonio Portuondo, y una contribución de Enrique Lihn sobre la novela Zoom de Hernán Valdés. En la sección 'Reseñas' se incluyen reseñas de Antonio Skármeta sobre Exceso de Mauricio Wacquez y de Raquel Olea sobre Los Buenos Días de Omar Lara y sobre Muerte y Maravillas de Jorge Teillier. Cabe agregar que un alto número de estos autores, tanto los que escribieron los artículos como aquellos cuyas obras se abordan en estos estudios, tuvieron que partir al exilio durante la dictadura militar y que sus libros desaparecerán de las librerías en Chile.

Como señalábamos, el panorama literario cambia drásticamente en el número 7, publicado - tras una pausa de tres años-en diciembre 1976, por cuanto desaparecen todos los nombres que pudieran ser vinculados con el proyecto político o cultural de la Unidad Popular. No obstante, este número 
permite también otra lectura: esto es, leerlo como un testimonio de que la literatura comprende un potencial que aun en los "tiempos más oscuros", al decir de Bertolt Brecht, le es inalienable y que en este caso se presenta como un humanismo general, como "experiencia vital", según lo formula Irma Céspedes en su estudio sobre Los cuentos de Canterbury. Esto se expresa en el hecho de que ahora la revista se aboca al estudio de autores del canon de la literatura mundial, tales como Chaucer, Gabriela Mistral, Goethe, Cortázar, Neruda, Juan Rulfo, Leopoldo Lugones y que los métodos aplicados por los académicos garantizan la despolitización de las lecturas. Se orientan principalmente por la narratología y por el estructuralismo francés (Todorov). Entre los/las colaboradores/as se encuentran cuatro colegas del Departamento de Literatura, un autor de la Pontificia Universidad Católica de Chile y dos autores de universidades o instituciones extranjeras (Academia de Lengua de Argentina y Universidad de California (Irvine)).

Asimismo, el número 8 (abril de 1977) continúa la tendencia de una 'internacionalización' temática con artículos sobre "El surrealismo y la literatura iberoamericana" de Cedomil Goic, sobre el Poema del mío Cid, primer texto épico español, de Hugo Montes, sobre "El Fausto de Goethe y del don Juan hispano" de María Isabel Sáenz-Villarreal S., un estudio sobre Arthur Rimbaud de Carlos Morand, un artículo de Ramón Suárez Mardones sobre la novela $L$ 'inquisitoire del autor francés Robert Pinget, novela que se inscribe en la tendencia del 'Nouveau roman', y una nota de María Eugenia Góngora sobre el autor medieval anónimo y su texto "Vida del poeta y trovador Jaufré Rudel", con una traducción de una de sus poesías del provenzal al castellano. En cambio, la literatura chilena se incluye solamente a partir de dos trabajos sobre poemas de Vicente Huidobro (Ana María Cuneo y Lucía Invernizzi). Nos encontramos entonces con un número que integra más trabajos sobre literatura europea que de literatura latinoamericana. Los tres artículos sobre esta última tratan de la vanguardia - con certeza una tendencia literaria no precisamente predilecta para aquellos sectores de la burguesía chilena proclive a la dictadura militar-. Sin embargo, esta internacionalización es ambivalente: por un lado, es una posibilidad de obviar un conflicto político e ideológico con la dictadura militar, por el otro, da pie a la internacionalización y las tentativas de integración cultural en el mundo occidental impulsado por el régimen militar.

Esta tendencia hacia la internacionalización se mantiene en los números 9/10 (agosto y diciembre 1977), publicados en un tomo. Destacan allí un artículo de índole teórica sobre la ironía literaria de Carmen Foxley, dos artículos 
sobre literatura chilena (uno sobre el "Poema de Chile" de Gabriela Mistral escrito por Iván Carrasco y otro sobre el drama de Luis Alberto Heiremans por Eduardo Thomas). Además, este volumen incluye cinco contribuciones sobre literatura europea: sobre poesía española (Fray Luis de León y García Lorca), literatura francesa (Claude Ollier y el 'nouveau roman') y literatura italiana (Dante). En tanto, en el número 11 no se publican trabajos sobre literatura chilena sino dos artículos sobre literatura alemana (Eichendorff y un estudio comparado entre Rilke y Vicente de Aleixandre), un análisis estructural de Pedro Páramo y una bibliografía extensa sobre "Estudios de Literatura Hispanoamericana en los Estados Unidos" de Jorge Román-Lagunas. En el siguiente número, $\mathrm{el} \mathrm{N}^{\circ} 12$, se incluye un artículo sobre literatura chilena, una entrevista con Miguel Arteche sobre su poesía y la poesía de su generación, realizada por Juan Villegas, quien comenta con cierto asombro el hecho de que a Arteche y los poetas alrededor de él en los años 50 no les importaba "lo que ocurría en el mundo" $(R C h L 12,54)$.

En conclusión, en los años bajo la dictadura militar la $R C h L$ se transforma definitivamente en una Revista de Literatura con un porcentaje alto de estudios dedicados a la literatura hispanoamericana, en particular a sus grandes autores consagrados -Rulfo, Borges, Vallejos-y a la literatura europea, especialmente española, pero también francesa y alemana. Las metodologías preferidas son de cuño estructuralista y comparatista. Por ende, puede inferirse que la preferencia por las 'literaturas del mundo' aparece como una estrategia para evitar temáticas y autores chilenos, los que, dadas las circunstancias, quiérase o no, implicarían un posicionamiento político. No obstante, en algunos casos es posible detectar ciertas alusiones de modo muy sutil a lo político, como por ejemplo en la cita de la entrevista con Miguel Arteche. La ya mencionada ambigüedad que implica la apertura a lo universal se hace evidente también en la reseña de la novela Casa de Campo de José Donoso escrita por Carlos Morand en el número 14 (1979). Allí, el autor, refiriéndose a otra novela de Donoso, El lugar sin límites, comenta: “[... ] aunque la historia se desarrolla en un ambiente típicamente chileno, todo lo que hay en ella de vernáculo funciona como un elemento adjetivo en un proyecto transcendente de componer algo de alcance más universal" ( $R C h L 14,151)$. En este mismo sentido interpreta también la novela Casa de Campo cuando la caracteriza como

[...] una extensa y barroca metáfora del mundo burgués, sostenida por el andamiaje hegeliano que explica la historia del hombre como un movimiento provocado por el encuentro de fuerzas antagónicas. Todo lo cual ha sido puesto en clave, una clave que es necesario de 
descifrar, pero que una vez descifrada, la novela pierde algo de su indudable encanto (152).

Esta lectura de la novela es una propuesta universalista que elude toda alusión a la dictadura militar -a no ser que se quiera interpretar la observación final de la cita como una alusión con este sentido, al decir que 'descifrar' la novela, si bien es 'necesario', le restaría parte de su 'encanto'. En otras palabras, se postula que la novela tendría una dimensión oculta, que alude a la realidad chilena. El crítico no lo dice directamente, pero su texto permite esta lectura, aun más, si tomamos en consideración sus referencias a la "enajenación" brechtiana, una técnica que -como es sabido- para Brecht siempre tuvo la función de revelar la realidad concreta en la cual vivimos.

No obstante, lo señalado no quiere decir que la apertura hacia la literatura del mundo tiene -en nuestra opinión- solamente la función de apartar a los lectores de la realidad política chilena. Con certeza, se le puede asignar también la función de proyectar lo vivido en Chile hacia un contexto universal, con el propósito de quitar a los acontecimientos experimentados su singularidad. Como lo sostiene Miguel Arteche en un comentario sobre su poema "El joven torturado" (escrito en 1967, provocado por un acto de tortura de la policía franquista española): “[...] ese poema vale, desde luego, no por su base real, sino porque alcanza a cualquier persona que haya sido torturada en el mundo, que esté siendo torturado o que vaya a ser torturada, ¿verdad?" ( $R C h L, 12$ 53). Las referencias a la condición humana en la situación chilena concreta de estos años nos confronta en diversos artículos con ejemplos literarios impresionantes, en los cuales se reflejan extremos de la condición humana como es el caso del artículo titulado "Poesía de Pierre Jean Jove" de Ramón Suárez $(R C h L 15)$ y sus otros artículos sobre literatura de lengua francesa (como por ejemplo su excelente trabajo sobre el teatro de Maeterlinck, en el número 20 de 1982), o la interpretación de una poesía de Georg Trakl y su comparación con dos traducciones al español publicada también en el número 15 de 1980.

Durante los años 80 la $R C h L$ va a mantener este tono y este horizonte internacional ${ }^{1}$. Aparte del reconocimiento de los esfuerzos que implica mantener 
una revista de literatura bajo estas circunstancias políticas, es importante también destacar la regularidad de su publicación y el hecho de que durante todo este periodo solamente en los años 1980 y 1986 dos números aparecen reunidos en un mismo tomo.

A partir de la mitad de la década de 80 , la $R C h L$ comienza a abrirse nuevamente a la literatura chilena actual. Inicialmente este proceso se evidencia en las reseñas. En el número 26 (1985) aparece una reseña de la novela Nina Asturriaga (Barcelona: Argos Vergara S.A. 1984) del autor Vicente Urbistone, en la que se lee: "Nina Asturriaga es el personaje que da el título a la novela, y que ocupa el centro de la enigmática y borrosa escena del acontecer social e histórico que se despliega entre el gobierno del Frente Popular y el de la Unidad Popular en Chile de los años treinta y ocho a setenta y dos" (145).

Empieza, pues, a superarse la "tabuización" de la Unidad Popular y del exilio. Lo que vale también para la nota de Walter Hoefler (RChL 27-28 de 1986) sobre "El (des)orden del peep-show de Schopf", texto publicado en Ediciones Manieristas, Santiago 1985, un libro de un autor que en este momento aún estaba en el exilio.

No obstante, como era de suponer, el cambio decisivo y significativo se puede constatar a partir de 1988. Encontramos en el número 32 (nov. 1988) dos reseñas, una sobre Poesía prisionera de Bruno Serrano (Santiago de Chile, 1988) y otra sobre Mi rebeldía es vivir de Arinda Ojeda Aravena (Concepción, 1988). Poesía prisionera (Escritura de cinco mujeres encarceladas) es el resultado notable de un trabajo de taller que el poeta Bruno Serrano llevó a cabo en 1987 con prisioneras políticas, el que significó, como dice el autor, un "Arduo esfuerzo para convencer en un principio a las autoridades, luego para atravesar la censura y a la vez conquistar los silencios negros que impone la

veces; Cedomil Goic, citado 14 veces; Wolfgang Kayser, citado 13 veces; Claude Bremond, citado 11 veces; Roman Jakobson, Félix Martínez y Martin Heidegger, citados 10 veces cada uno; E. R. Curtius, citado 9 veces; Jacques Derrida, Paul Ricoeur y Ramón Menéndez Pidal, citados 8 veces cada uno; José Ortega y Gasset citado 7 veces; Gerard Genette y A.J. Greimas, citados 6 veces cada uno; Umberto Eco y Georg Lukács citados 5 veces cada uno; Emir Rodríguez Monegal, François Recanati y Emile Benveniste, citados 4 veces cada uno; y Julia Kristeva, Phillipe Hamon y C.G. Jung, citados 3 veces. Las obras más citadas son Interpretación y análisis de la obra literaria de W. Kayser, con 11 menciones, La estructura de la obra literaria de F. Martínez, con 10 menciones y Literatura europea y edad media latina de E.R. Curtius, con 8 menciones" (137). 
autocensura" (159). Por su parte, Arinda Ojeda, autora de Mi rebeldía es vivir, era en este momento prisionera política en la Cárcel de Coronel (cfr. 159).

En ese mismo número se publica una entrevista de Verónica Cortínez con Antonio Skármeta e Isabel Allende, la que se realiza en Houston, Texas, en el mes de abril de 1988. En ella, Skármeta constata que su evolución como escritor "ha estado muy ligada a mi biografía personal y a mi biografía colectiva" [...] "La segunda etapa (de esta biografía) me viene impuesta desde fuera, de una manera violenta, con el golpe" (81). Por su parte, Isabel Allende responde a la pregunta si "la literatura chilena escrita dentro de Chile y la del exilio tienen una base común, una continuidad": "Yo siento que esa división de los de adentro y afuera es un invento de la dictadura. Los que estamos afuera no estamos afuera porque nos dé la gana, sino porque no podemos vivir en una dictadura o porque nos echaron" (87).

Es a partir de estos números que el exilio, la dictadura militar y sus crímenes contra los derechos humanos no serán más temas "tabuizados" en la $R C h L$. Ahora la Revista se abre también hacia la poesía escrita durante la dictadura que trata de las experiencias vividas bajo el poder de este régimen. Uno de los textos más relevantes en este contexto es Carta (sic) de prisionero de Floridor Pérez publicada en el número 35, en abril de 1990 (47-77). No obstante, la interpretación de los poemas de Carta (sic) de prisionero que realiza Ana María Cuneo es aún un ejemplo de cómo la elección de un método de interpretación, en este caso la intertextualidad, permite soslayar el contexto concreto en el cual los poemas fueron escritos y al cual se refieren.

\section{RECONCILIACIÓN SEGÚN SAN MATEO}

$\mathrm{Al}$ teniente $\mathrm{K}$.

Su diestra

arrancaba mechones

rompía pómulos

diestra

$\mathrm{Su}$ diestra

quemaba con cigarros

hería con agujas,

siniestra

Tiempo después

-noticia siniestra-

con una granada 
se voló la diestra.

$\mathrm{Si}$ ahora cristianamente

yo le pusiera mi otra mejilla usted tendría que golpearme con la otra mano.

En su análisis de este poema, Ana María Cúneo señala: "Intertextualidad y bisemia son los procedimientos que rigen el desarrollo de este poema" (71). Se mencionan también los "presos políticos" (70) y la tortura (70-72), no obstante, en la conclusión se insiste: “"Carta (sic) de Prisionero' es un libro de poemas que entrega numerosas claves del quehacer de unos hombres cuyas vidas y cuya escritura se pueden ubicar en esa misteriosa zona que se despliega entre luces y tinieblas" (77).

La fase política y cultural de la llamada "transición a la democracia" a partir de 1990 se refleja también en la $R C h L$. No obstante, en comparación con la ruptura radical que significó el golpe militar de 1973 para la vida política y cultural del país, los cambios producidos por la "transición" fueron mucho más lentos. Ello vale también para la Revista, cuyo perfil se redefinirá paulatinamente a partir de esta década. Responsables de estos cambios no son solamente la transformación del país y los factores internos asociados, sino también procesos como la internacionalización de las universidades y de las ciencias humanas, como consecuencia del impacto de la entonces incipiente era digital. Las nuevas posibilidades técnicas, el computador personal y el envío de manuscritos por vía digital facilitan la producción de textos y aceleran su circulación. El proceso engorroso de los textos mecanografiados con la máquina de escribir y sus limitadas posibilidades de reproducción fue sustituido por las fotocopiadoras a partir de los años 70, facilitando la multiplicación de los textos. En los años 90, con el uso generalizado del PC, se desarrollan nuevas técnicas de escritura (la escritura en la pantalla, la simplificación de las correcciones) las que tienen un impacto profundo en la elaboración de los manuscritos. La posibilidad de enviar los textos en forma digital (una corta fase intermedia fue la tecnología del Fax) significó la aceleración vertiginosa de las comunicaciones y los procesos de difusión y contactos internacionales. En el caso particular de Chile, ello significó para sus intelectuales la rápida superación de nuestro proverbial aislamiento insular. De pronto, los contactos e intercambios son tan rápidos como desde Madrid, Pittsburgh o Frankfurt, y al revés, textos escritos en Chile llegan en tiempo real a cualquier parte del mundo. 
Paralelamente, la progresiva digitalización de los textos abre un amplio espectro de posibilidades de darse a conocer y de acceder a publicaciones a nivel universal a través de la 'world wide web', y la cercanía física a grandes bibliotecas no es ya una ventaja decisiva para el trabajo intelectual. Todos estos factores contribuyeron a un crecimiento significativo de la cantidad de publicaciones científicas, especialmente en las Humanidades, $\mathrm{y}$, en consecuencia, a la reestructuración de revistas existentes así como a la creación de nuevas publicaciones.

En este contexto cambió también el perfil de la $R C h L$ y los procesos de su funcionamiento académico administrativo. En tiempos de su creación, a comienzos de los 70, la $R C h L$ se pensó primordialmente como un órgano de difusión y reflexión del quehacer departamental y un medio de difundir las investigaciones de sus académicos, con la intención de posicionarse y hacerse escuchar en el contexto del debate nacional. Tras el interregno de la dictadura a partir de los años 90, la Revista modifica paulatinamente su perfil en tanto, si bien mantiene su condición de ser un órgano de publicación para los académicos del Departamento de Literatura ${ }^{2}$, crecientemente su énfasis se centrará en la disciplina y la difusión de las investigaciones que se realizan bajo su alero tanto en Chile como a nivel internacional. Consecuentemente, el Comité de Redacción asume funciones cada vez más específicas: será responsable de la revisión de las colaboraciones recibidas y asegurar la calidad científica de los artículos a publicar, estará a cargo de la coordinación de las evaluaciones ciegas por los pares evaluadores nacionales e internacionales, cuyos informes pasan a ser el criterio primordial de selección de los artículos que finalmente se publican.

\footnotetext{
Cabe pensar varios motivos para explicar la disminución de la presencia de los académicos del Departamento de Literatura: en las revistas indexadas, como la $R C h L$, solamente un porcentaje limitado de los artículos puede ser escrito por los científicos de la institución. Por otra parte, los artículos, resultados de proyectos de investigación financiados por Fondecyt deben, de preferencia, ser publicados en revistas que no pertenecen a la institución del investigador, es decir, los colegas del Departamento en esa situación no publican en la $R C h L$. Y, por último, la decisión individual de los académicos de publicar sus trabajos "fuera de la casa" en revistas de otras universidades, de preferencia de otros países. Ello es también reflejo del grado de internacionalización del Departamento, sus contactos con universidades de otros países, la participación de sus académicos en eventos internacionales con la probabilidad y la obligación de que un número importante de trabajos de nuestros colegas en los últimos años no aparezcan en esta revista.
} 
Por otra parte, a partir de 2012 , la $R C h L$ será publicada paralelamente en una versión en papel y en una versión digital, lo que la hace accesible sin restricciones globalmente. En 2016 se completa la digitalización de todos sus números, permitiendo el acceso desde cualquier lugar y en cualquier momento a todos sus números desde su fundación en 1970. Asimismo, la incorporación de resúmenes y "palabras clave" en el diseño de cada artículo publicado, ha facilitado su incorporación más informada a las bases de datos digitales y su utilidad en los procesos de búsqueda o consulta. Adicionalmente, la era de la digitalización nos ofrece también cifras sobre la difusión nacional e internacional de la $R C h L$ : entre los años 2014-2018 suma cerca de 630 mil visitas a su sitio web ${ }^{3}$.

Como ya se señaló, la transformación del perfil de la revista a partir de los años 90 fue el resultado de las tendencias de internacionalización como también del proceso político de la transición. Poco a poco la $R C h L$ da señales contundentes de sus nuevos compromisos con la vida literaria y la cultura chilena actual, sin renunciar al horizonte internacional que ha conquistado durante los años de la dictadura, - una alternativa, como se dijo, ante la necesidad de evitar exponerse a eventuales censuras. Se hace cargo de nuevos temas (v. gr., feminismo ${ }^{4}$, escritura de mujeres ${ }^{5}$, poesía mapuche ${ }^{6}$, drama y teatralidad $\left.{ }^{7}\right)$, discute nuevas perspectivas teóricas y metodológicas (v.gr., estudios de género, estudios poscoloniales, teorías del discurso, entre

3 Estas cifras se distribuyen de manera siguiente: Chile 38\%; Argentina 14\%; México 12\%; Colombia 10\%; Perú 8\%; Ecuador 4\%; Venezuela 2\%; EE.UU. 5\%; España 10\%. Estas cifras no consideran la recepción de los $c a .250$ ejemplares de cada número que circulan en papel. Fuente: Dirección de Servicios de Información y Bibliotecas (SISIB) (2019). "Informe Revista Chilena de Literatura. Proyecto CONICYT FP180001".

4 Cfr. Kemy Oyarzún. "Género y etnia: Acerca del dialogismo en América Latina" (RChL 41, 1993).

En sintonía con la tendencia internacional aumentó tanto el porcentaje de académicas femeninas que publican en la revista como también de escritoras a las cuales se dedican los artículos. Entre los años 1970 y 1985, 16\% de los artículos fueron escritos por mujeres mientras que entre 2008 y 2018 el porcentaje de artículos de autoras subió a 36\%. Fuente: Dirección de Servicios de Información y Bibliotecas (SISIB) (2019). Informe Revista Chilena de Literatura. Proyecto CONICYT FP180001.

$6 \quad$ Cfr. Iván Carrasco. "Las voces étnicas en la poesía chilena actual $(R C h L 47,1995)$ y "De la Tierra sin fuegos. Voz de los que no tienen voz" ( $R C h L 52,1998)$.

$7 \quad$ Cfr. el artículo "Panorama crítico de los hitos del drama chileno en la Revista Chilena de Literatura" de Carolina Brncić y Eduardo Thomas sobre esta temática, en este volumen. 
muchos otros $\left.{ }^{8}\right)$, artículos sobre autores chilenos hasta entonces vedados o recién emergentes (v.gr., Isabel Allende y Diamela Eltit ${ }^{9}$, Raúl Zurita o Pedro Lemebel).

No obstante, el cambio más significativo es el acercamiento crítico a los años de la dictadura militar y sus repercusiones en la literatura. Para nombrar solo algunos ejemplos, Carlos Cortínez publica en el número 51 de 1997 desde Florida A.M. University, un artículo sobre "El temblor poético de Trilce en el sur de Chile", donde resalta las consecuencias de la dictadura militar para esta revista ${ }^{10}$. Otro de los temas relevantes, asociados al golpe y los años de la dictadura, se vincula con los problemas de la memoria y la recuperación del pasado reciente, los exilios internos y externos, en la literatura chilena y latinoamericana. Un ejemplo temprano de esta línea temática es el trabajo de Pércio B. de Castro (University of Dayton) ( $R C h L$ $52,1998)$ quien analiza en la Nota "La muerte y la doncella: ¿de quién son las bolas? Opresor oprimido y viceversa" pieza dramática del autor chileno, Ariel Dorfman, y las reflexiones implicadas sobre la tortura, el trauma de los torturados -en este caso una mujer-y cuestiones éticas apremiantes de cómo tratar y convivir con los ejecutores de estos crímenes.

$8 \quad$ Al respecto, cfr. David Wallace y Sergio Carumán "El rol de la teoría literaria en la Revista Chilena de Literatura: estudio diacrónico-descriptivo y crítico de los noventa y ocho números publicados", en este volumen.

9 Rodrigo Cánovas, "Una reflexión sobre la novelística chilena de los años 80 " ( $R C h L$ 38, 1991). Respecto a las novelas de estas autoras, Cánovas sostiene: "Estas novelas corresponden a dos paradigmas que se oponen y se complementan. A nivel histórico, una apuesta a una continuidad de las tradiciones nacionales; la otra anota la ruptura; a nivel femenino, una imagina a la mujer junto al hombre, la otra juega a prescindir de él; y, a nivel escritural, el gesto comunicativo amplio de Isabel Allende se corresponde con la transgresión vanguardista en Diamela Eltit. Los novelistas chilenos no solo dialogan con las tradiciones de la nueva narrativa hispanoamericana sino, también, con la tradición literaria chilena" (105).

$10 \quad$ Al respecto dice Cortínez: "Ahora bien, otro hecho importante en la historia de Trilce, aunque trágica en el contexto nacional, fue el golpe militar que, en septiembre de 1973, pusiera fin a la democracia chilena. Se sabe que la acción militar de Pinochet intentó justificarse como reacción en contra de las fallas del gobierno de Allende, pero al irse al otro extremo, el nuevo gobierno cayó no solo en errores sino en el exceso, la tortura y el crimen, que son, por lo demás, lo habitual en regímenes dictatoriales. Los artistas e intelectuales chilenos fueron tratados con igual dureza que los combatientes políticos, asignándoseles a aquéllos la responsabilidad por las acciones de éstos. Así se produjo esa dolorosa diáspora que afectó a miles de chilenos, atenuada por la generosa hospitalidad que muchos países ofrecieron a los desterrados. La actividad poética de Trilce, aunque privada de medios en Chile, continuó en el exilio y tuvo cierto apoyo internacional" (103). 
Finalmente, si cabe caracterizar las últimas dos décadas de la $R C h L$ en relación con uno de sus rasgos más sobresalientes, este sería su gran diversificación teórico-metodológica, que "incluye una amplia gama de fenómenos textuales"11 que abarcan la Colonia, el siglo XIX y el siglo XX, pero sobre todo la literatura contemporánea, chilena y latinoamericana, aunque sin descuidar las literaturas europeas, incluyendo la española. Ante esta tendencia, parece una medida adecuada el que desde el número 96 (noviembre 2017) se haya incluido un dossier temático de entre cuatro y seis artículos en cada volumen. Esta práctica constituye una proyección de los esporádicos "números especiales" dedicados a un autor o una temática especial, como es el caso del número 79 (2011) dedicado a Pablo Neruda, el número 80 (2011) con un dossier extenso sobre Mario Vargas Llosa, el número 84 (2013), un volumen monográfico sobre las Humanidades, el número 85 (2013), un número también monográfico sobre Europa y América colonial, transmigraciones y diálogos, el número 88 (2014), centrado en literatura y cultura brasileña y el número 91 (2015) dedicado a estudios sobre Nicanor Parra.

Respecto a los criterios que rigen la selección de los artículos a publicar nos importa destacar que la $R C h L$ no favorece una línea teórica, metodológica o temática, sino que se orienta cabalmente por los principios establecidos en la "website" ${ }^{12}$ de la revista. En esta página se explicita: "La Temática de la Revista es amplia en el campo de la investigación literaria y de las Humanidades, en cuanto abarca el estudio de escritores y obras literarias y afines, tanto de Chile como del extranjero, de épocas anteriores o actuales, siempre desde una perspectiva literaria". Por otra parte, se exige de los artículos, estudios, notas y documentos, un aporte innovador al debate teórico y metodológico, o de manera más general, como dice en la cita, un aporte "en el campo de la investigación literaria y de las Humanidades". Esta suerte de declaración de principios tiene un doble alcance, estrictamente disciplinario, por una parte, pero también en relación a lo que podríamos precisar como inspiración ética o de crítica ideológica, dimensión que amerita una reflexión y contextualización más general.

11 Cfr. David Wallace y Sergio Carumán "El rol de la teoría literaria en la Revista Chilena de Literatura: estudio diacrónico-descriptivo y crítico de los noventa y ocho números publicados", en este volumen.

$12 \mathrm{https}: / /$ revistaliteratura.uchile.cl/index.php/RCL/index 
Las Humanidades son disciplinas crecientemente cuestionadas en nuestros días: desafiadas por las 'ciencias duras' y por las innovaciones técnicas, confrontadas con las exigencias cada vez más restrictivas de garantizar su rentabilidad y de convertirse en un producto del mercado, con la finalidad de asegurar su autofinanciamiento. No obstante, si revisamos la historia de "las Humanidades" en la época de la modernidad nos damos cuenta de que su existencia nunca ha sido fácil. Siempre han estado confrontadas a las opciones de pactar con el poder, sus autoridades y sus instituciones, o de perseverar en la libertad de un pensamiento independiente y crítico por el precio de correr el riesgo de ser reprimidas o por lo menos de verse privadas del apoyo de parte de las instituciones oficiales. En consecuencia, "las Humanidades" siempre han sido contradictorias en sí mismas: no solamente fueron portavoces de una modernidad inspirada en los principios de "libertad, igualdad y fraternidad" para todos, sino que fueron también defensoras de una modernidad que buscó imponerse a costa de la dignidad humana. En tal sentido, se vieron históricamente expuestas a ideologizaciones de las más distintas envergaduras. Defendieron la Ilustración y el sapere aude kantiano, sirvieron a la construcción de los Estados nacionales, los cuales después buscaron someterlas a su control, legitimaron la superioridad civilizatoria de las naciones europeas en la política colonizadora, ofrecieron sus servicios para los sistemas totalitarios del siglo XX, y hoy día no faltan corrientes que también se consideran "humanistas" defendiendo políticas culturales del neoliberalismo y neoconservadurismo. "Las Humanidades", una vez institucionalizadas, fueron frecuentemente enemigas decididas de "las Humanidades" independientes.

Por otra parte, si bien una función importante de las universidades ha sido siempre la de defender la independencia y la autonomía de "las Humanidades" y de ofrecerles un espacio para sus reflexiones críticas, no siempre han logrado cumplir con este objetivo. En este sentido, una tarea primordial de una Facultad de Filosofía y Humanidades es -en nuestra opinión- la de incentivar y apoyar la revisión crítica y pública de las propias Humanidades en un proceso de autorreflexión continua. Esta autorreflexión será su defensa más seria y eficaz para garantizar su actualidad y el cumplimiento de su misión ante la sociedad.

En este sentido, la literatura ha sido y continúa siendo con certeza una de las instancias privilegiadas en la cual la modernidad -o si se quiere, la emergente posmodernidad-, en sus manifestaciones más variadas, ha sido repensada en forma creativa y crítica. En el plano específico de los estudios 
literarios la autorreflexión será tanto más eficaz y lograda en la medida en que su objeto no se define solamente como manifestación de lo nacional sino como un espacio donde lo nacional se comprende y se expresa en sus variados y más diversos lazos con las culturas y las literaturas del mundo, de otras épocas, de otras tradiciones estéticas y poéticas, de otras formas de convivencia humana. El medio literatura siempre se ha alimentado de un acervo no puramente local o nacional, incluso si se concentra en temáticas o problemáticas de origen local o nacional. El medio literatura siempre ha asumido, o por lo menos debía hacerlo, una responsabilidad ética -aun en los casos donde lo ético se manifiesta solamente en la forma estética- que trasciende lo regional.

Un espacio privilegiado donde esta dimensión de lo literario es discutida y se hace patente son las revistas literarias. La progresiva e intensa internacionalización que se manifiesta en las últimas décadas en las revistas de literatura ha contribuido notablemente a su perfilamiento como espacio crítico de investigación y de reflexión sobre la y la(s) literatura(s) y su posicionamiento en estos procesos, sus compromisos con las Humanidades y con su responsabilidad de defender la dignidad humana. La $R C h L$ comparte esta condición explícita o implícitamente como lo evidencia la revisión de su historia y la variedad de sus respuestas a estos desafíos, así como su creciente participación en este diálogo cada vez más global. Un diálogo cuyo lugar de enunciación será siempre desde Chile, pero nunca restringiendo sus miradas a los límites de este país. Ahora bien, en qué medida ella ha cumplido con su tarea de ser una revista representativa de una Facultad de Filosofía y Humanidades y su misión en la sociedad es una interrogante a responder por sus lectores.

\section{BIBLIOGRAFÍA}

Águila, Elena y Luis Correa Díaz. "Bruno Serrano. Poesía prisionera y Arinda Ojeda Aravena. Mi rebeldía es vivir". Reseñas. Revista Chilena de Literatura 32 (1988): 158-160.

Benedetti, Mario. “Imitación de Propercio”. Fragmento de Apocalipsis, en: Fernández, Mario Rodríguez: "La poesía de Ernesto Cardenal". Revista Chilena de Literatura 1 (1970): 87-89.

Brncić, Carolina y Eduardo Thomas. "Panorama crítico de los hitos del drama chileno en la Revista Chilena de Literatura". Revista Chilena de Literatura 100 (2019): 139-170.

Cánovas, Rodrigo. "Una reflexión sobre la novelística chilena de los años 80". Revista Chilena de Literatura 38 (1991): 101-108. 
Carumán, Sergio y David Wallace. "El rol de la teoría literaria en la Revista Chilena de Literatura: estudio diacrónico-descriptivo y crítico de los noventa y ocho números publicados". Revista Chilena de Literatura 100 (2019): 35-94.

Castro, Pércio B. De. "La muerte y la doncella: ¿de quién son las bolas? Opresor oprimido y viceversa”. Revista Chilena de Literatura 52 (1998): 61-67.

Cortínez, Carlos. "El temblor poético de Trilce en el sur de Chile". Revista Chilena de Literatura 51 (1997): 99-115.

Cortínez, Verónica. "Polifonía: Entrevista a Isabel Allende y Antonio Skármeta". Revista Chilena de Literatura 32 (1988): 79-89.

Cuneo, Ana María. "Carta (sic) de prisionero de Floridor Pérez". Revista Chilena de Literatura 35 (1990): 47-77.

Dirección de Servicios de Información y Bibliotecas (SISIB). "Informe Revista Chilena de Literatura. Proyecto CONICYT FP180001”, 2019.

Foxley, Carmen. "Vicente Urbistondo. Nina Asturriaga". Reseña. Revista Chilena de Literatura 26 (1985): 145-147.

Hoefler, Walter. "El (des)orden del peep-show de Schopf". Revista Chilena de Literatura 27-28 (1986): 209-212.

Morand, Carlos. "Casa de Campo de José Donoso". Reseña. Revista Chilena de Literatura 14 (1979): 151-152.

Parra, Nicanor. "Palabras obscenas". Revista Chilena de Literatura 1 (1970): 79-85.

Rosenfeld, Corina. "La Revista Chilena de Literatura: 1970-1985". Revista Chilena de Literatura 38 (1991): 127-138.

Villegas, Juan. "Entrevista a Miguel Arteche”. Revista Chilena de Literatura 12 (1979): 41-54. 